\title{
Geochemistry of Soil Nanoparticles
}

\author{
Panova $\mathrm{EG}^{1 *}$, Oleynikova $\mathrm{GA}^{2}$, Matinian $\mathrm{NN}^{1}$ and Bahmatova $\mathrm{KA}^{1}$ \\ Department of Geochemistry, Institute of Earth Science Saint-Petersburg State University, Russia
}

Submission: January 13, 2018; Published: February 05, 2018

*Corresponding author: Panova EG, Department of Geochemistry, Institute of Earth Science Saint-Petersburg State University, University Enb. 7/9, 199034, Saint-Petersburg, Russia, Tel: +7(921)4354881; Email: elena-geo@list.ru

\section{Mini Review}

One of fundamental problems of soil science is studying of natural mechanisms of metals behavior in a pedosphere that has both scientific, and applied value. Thus the most important part is assigned to mobile migratory and capable forms of chemical elements.

It has been shown that soil colloids play an important role in existence and functioning of the soil and participate in migration of chemical elements. Colloidal particles (the size less than 1000 nanometers) play an important role not only by elements transport on a soil profile; they can react with a mineral matrix and control availability of chemical elements [1-5].

The fact of interaction of organo-mineral colloids with soils micro-particles was noted in the middle of the last century, but only at the present time became possible to reveal fractal structure of organo-mineral soil gels to create colloidal and chemical model of the soil [3].

There are some methods for allocation of soil colloids: centrifugation methods, expression and replacement were used; for selective extraction of any components of a soil - a leaching method. However addition of chemical reagents for extraction can lead to emergence of artifacts, there is a lot of reasons for which emergence.

Modern analytical equipment and methods of direct visual supervision nano-objects allowed to distinguish particles up to 0.25 nanometers in size. However definition of a chemical composition of nanoparticles causes certain difficulties. When carrying out electron probe researches because of their ultra small size of particles the probe with a diameter of $1 \mathrm{mk \mu}$ burns them through that doesn't allow to estimate their chemical composition separately from a matrix. Thus limits of detection of chemical elements are at the level of $0.01 \%$ that a priori excludes from the analysis sphere the majority of microcells. It is necessary to consider also that the local structure of separate particles of test doesn't reflect geochemical specifics of a sample [6].
The colloidal fraction (nano-fraction) with a particles size less than $1000 \mathrm{~nm}$ is taken by water from a soil by water under specially picked up conditions. The nano-fraction represents part of test in which chemical elements are in ionic, molecular and colloidal forms, and concluded in soil inter-grain space water colloidal and salt solution. Thus, the more steam space of a sample, the higher in it a nano-fraction share (to the 6 weight. $\%)$. Soil samples and its nano-fraction were analyzed by the ICP MS method. It is necessary to point out, that analysis of water solutions allow to realize whole possibilities of ICP MS method at most, because there is no negative influence of additional chemical reagents which in its turn results in lowering the detection limits of rare and trace elements in initial samples in 2-3 orders (ppm): 0,0002 (Au); 0,0004 (Pt); 0,0006 (Pd); 0,0001 (Ag); 0,0001 (Rh); 0,0004 (Ru); 0,0001 (Ir); 0,0001 (Re); 0,00006 (Tl); 0,0006 (Cd); 0,0008(Hg); 0,002 (Te); 0,0002 (Sb); 0,001 (As); 0,0006 (Ge); 0,0002 (Ga); 0,0006 (Mo); 0,00002 (U); 0,01 (Se); 0,0001 (Bi); 0,0002 (In); 0,0006 (Sc) and so on (Oleynicova, Panova, 2007).

Coefficient of accumulation $(\mathrm{K})$ is calculated as the ratio of element concentration in nano-fraction to its concentration in the whole soil sample (Table 1). Especially considerable differences in the proportion of fixed and mobile forms of chemical elements were observed in the sandy soil, probably due to its greater pore space. In the clayey soil, the share of fixed forms of macro- and microelements was high; in the sandy soil, mobile forms predominated.

In all the horizons of the clayey soil, the share of this fraction is greater than in the sandy soil. This fact can be explained by the higher content of fine-dispersed particles, including the colloidal ones. In the sandy soil, the proportion of mobile chemical elements was high; in the clayey soil, their share was much lower. In both profiles, the most mobile were Sc, Zn, Mo, and Ag. In the clayey soil, the maximum contents in nano-fraction were confined to the $\mathrm{BC}$ and $\mathrm{C}$ horizons. The pattern of water-soluble element distribution in the sandy soil is more complicated. There were elements with maximum accumulation in the E (Sc, Ni, Co, $\mathrm{Pb}$, and $\mathrm{Cu}$ ) and humus horizons ( $\mathrm{Ag}$, $\mathrm{Mo}$, and $\mathrm{Zn}$ ). 


\section{Conclusion}

A method for the isolation of the nano-fraction for analyzing the mobility of chemical elements is suggested. The analysis of water solutions without decomposition of soil and addition of reagents allows maximizing the opportunities of mass spectrometry with inductively coupled plasma (to lower the detection level for rare and scattered metals, to increase the spectrum of elements, and to get reliable information at their super-low concentrations), which makes it possible to raise significantly the information capacity of soil-chemical studies.

The portions of easily and difficultly mobile forms of elements were evaluated for two soils of different geneses in the Russian Plain. Significant differences in the total content of chemical elements (based on the total chemical analysis) in the soils and their water-soluble fraction, as well.

The course of ecological-geochemical research the analysis of nano-fractions give the opportunity to evaluate the behavior of water soluble (mobile) forms of chemical elements which can change essentially the quality of ecological investigations for possible effects of polluting by toxicants.

The work has been done in the chemical laboratory of the All Russian geological Institute and the in Resource centers of SPBU "Geomodel" and "Methods of analysis of the substance"

\section{References}

1. Amrhein C, Mosher PA, Strong JE (1993) Colloid-assisted transport of trace metals in roadside soils receiving deicing salts. Soil Sci Soc Am J 57(5): 1212-1217.

2. Cances B, Ponthieu M, Casterc-Rouelle M (2003) Metal ions speciation in a soil and its solution. Geoderma 113: 341-355.

3. Fedotov GN, Dobrovolskiy GV (2006) Colloid chemical model for describing some soil processes. Eurasian Soil Sci 39(5): 477-485.

4. Oleinikova GA, Panova EG (2007) Information resources of analysis of nanofractions of soils. Vestn S-Peterb Gos Univ No 3: 60-66.

5. Shein EV, Devin BA (2007) Current problems in the study of colloidal transport in soil. Eurasian Soil Sci 40(4): 399-408.

6. Wilson MA, Tran NH, Milev AS, Volk H, Max Lu GQ (2008) Nanomaterials in soils. Geoderma 146: 291-302. 